BNL- 75105-2005-CP

\title{
Synchrotron Radiation Monitor for NSLS Booster
}

\author{
I. Pinayev and T. Shaftan \\ NSLS, Brookhaven National Laboratory, Upton, NY
}

\author{
National Synchrotron Light Source \\ Brookhaven National Laboratory \\ P.O. Box 5000 \\ Upton, NY 11973 \\ www.bnl.gov
}

Notice: This manuscript has been authored by employees of Brookhaven Science Associates, LLC under Contract No. DE-AC02-98CH10886 with the U.S. Department of Energy. The publisher by accepting the manuscript for publication acknowledges that the United States Government retains a non-exclusive, paid-up, irrevocable, worldwide license to publish or reproduce the published form of this manuscript, or allow others to do so, for United States Government purposes. 


\section{DISCLAIMER}

This report was prepared as an account of work sponsored by an agency of the United States Government. Neither the United States Government nor any agency thereof, nor any of their employees, nor any of their contractors, subcontractors or their employees, makes any warranty, express or implied, or assumes any legal liability or responsibility for the accuracy, completeness, or any third party's use or the results of such use of any information, apparatus, product, or process disclosed, or represents that its use would not infringe privately owned rights. Reference herein to any specific commercial product, process, or service by trade name, trademark, manufacturer, or otherwise, does not necessary constitute or imply its endorsement, recommendation, or favoring by the United States Government or any agency thereof or its contractors or subcontractors. The views and opinions of authors expresses herein do not necessarily state to reflect those of the United States Government or any agency thereof. 


\title{
Synchrotron Radiation Monitor for NSLS Booster
}

\author{
I. Pinayev and T. Shaftan, NSLS, BNL
}

\section{Introduction}

NSLS booster diagnostics consisted of tune measurement system, system for turnby-turn measurement on the electron beam, and beam intensity monitor, which is not absolutely calibrated. We present design and implementation of synchrotron light monitor for the booster, which expands diagnostics capabilities. The system allows to measure an orbit, beam sizes and coupling of the electron beam along the ramp.

\section{Set-up}

There are eight synchrotron radiation ports available for diagnostics. We chose the most accessible one, which is close to the achromatic bend cave. The layout of synchrotron radiation monitor is shown in Fig. 1. Synchrotron light is emitted by the circulating electron beam in the middle of B4BM1 magnet. An aperture, installed immediately after output window, cuts off the radiation scattered from the walls of booster vacuum chamber. Light passes through an iris, which is used for precise control of the light intensity and is reflected up by a broadband metallic mirror. A lens with 30 $\mathrm{cm}$ focal length creates image of the electron beam with demagnification of 5.7. Coarse intensity control is performed by a filter wheel, which has six positions. Five positions are filled with neutral density filters with optical density varying from 1.0 to 5.0, while the sixth position is left empty. The wheel has remote control, which allows adjustment of the image intensity from the NSLS control room. View of the whole installation one can find in Fig. 2.

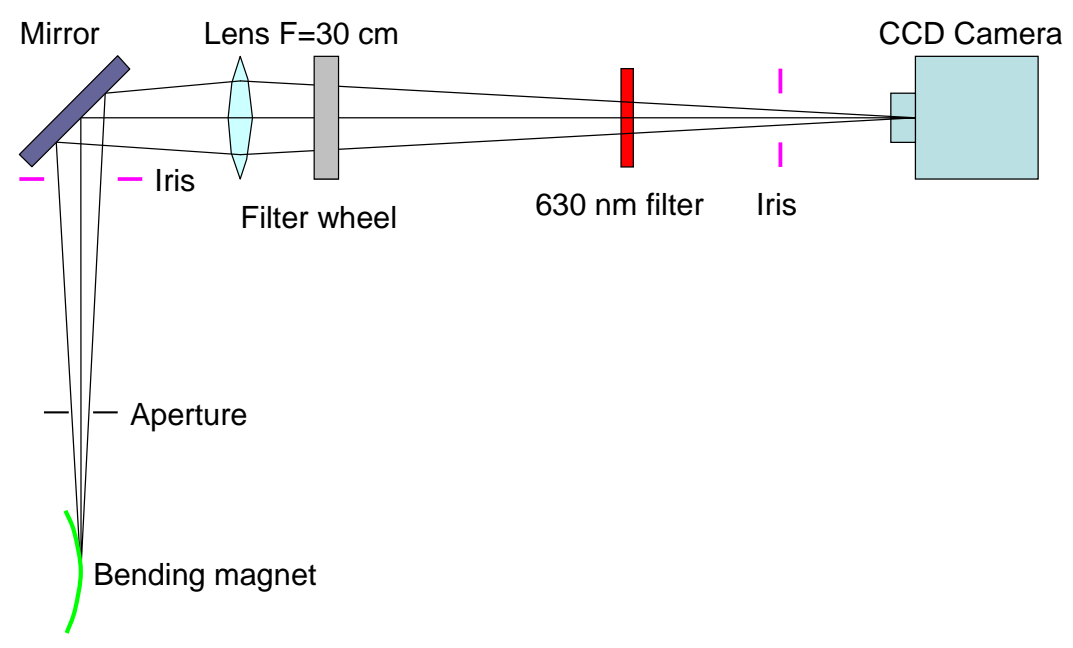

Figure 1. Present layout of synchrotron radiation monitor for booster. 
Over the ramping cycle the critical wavelength of the synchrotron radiation changes from 6.87 microns to 28 nanometers (see Fig. 3). To decrease intensity changes associated with this effect we installed a narrowband optical filter with central wavelength of $630 \mathrm{~nm}$.

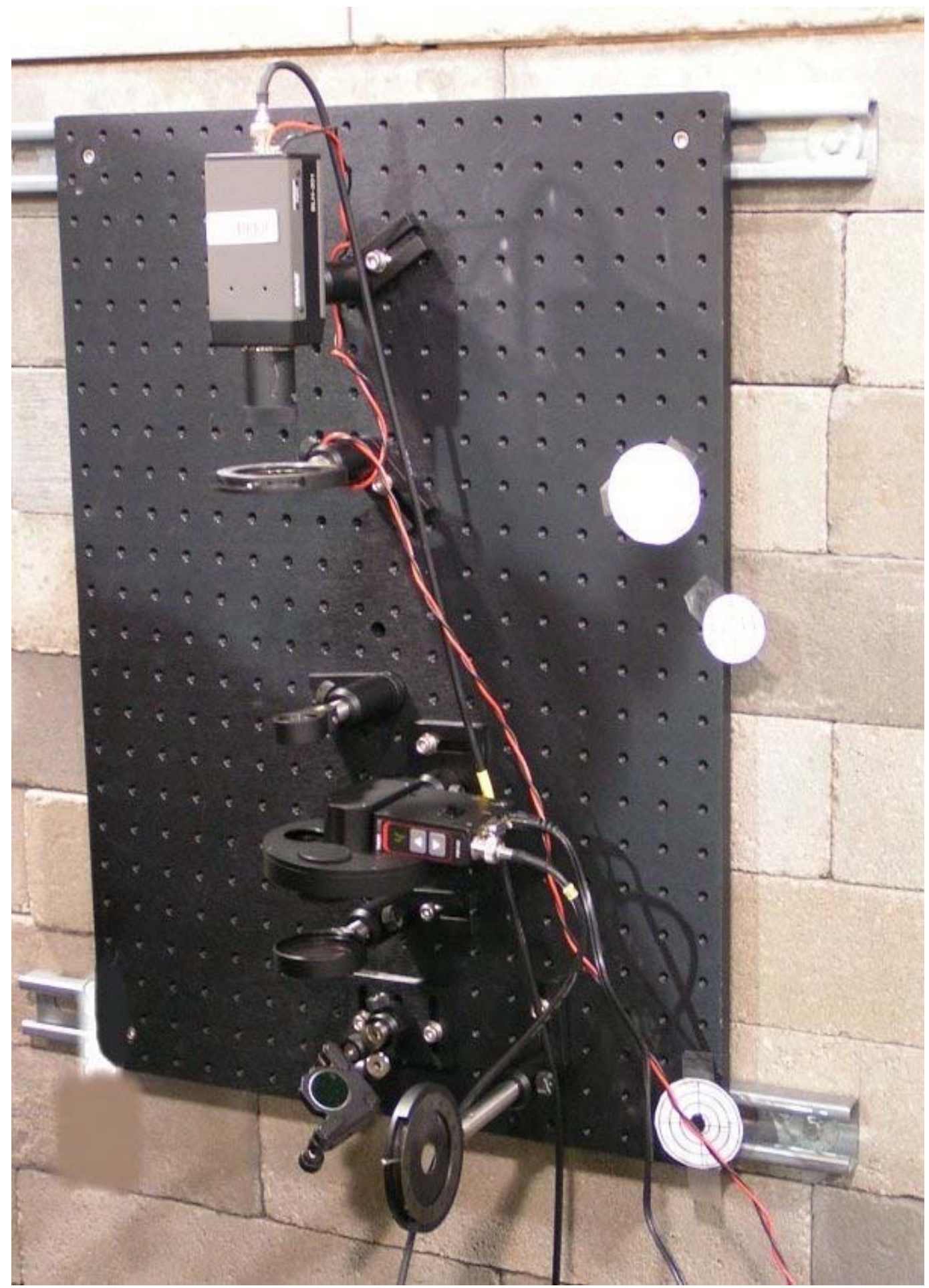

Figure 2. View on the synchrotron radiation monitor for booster. 


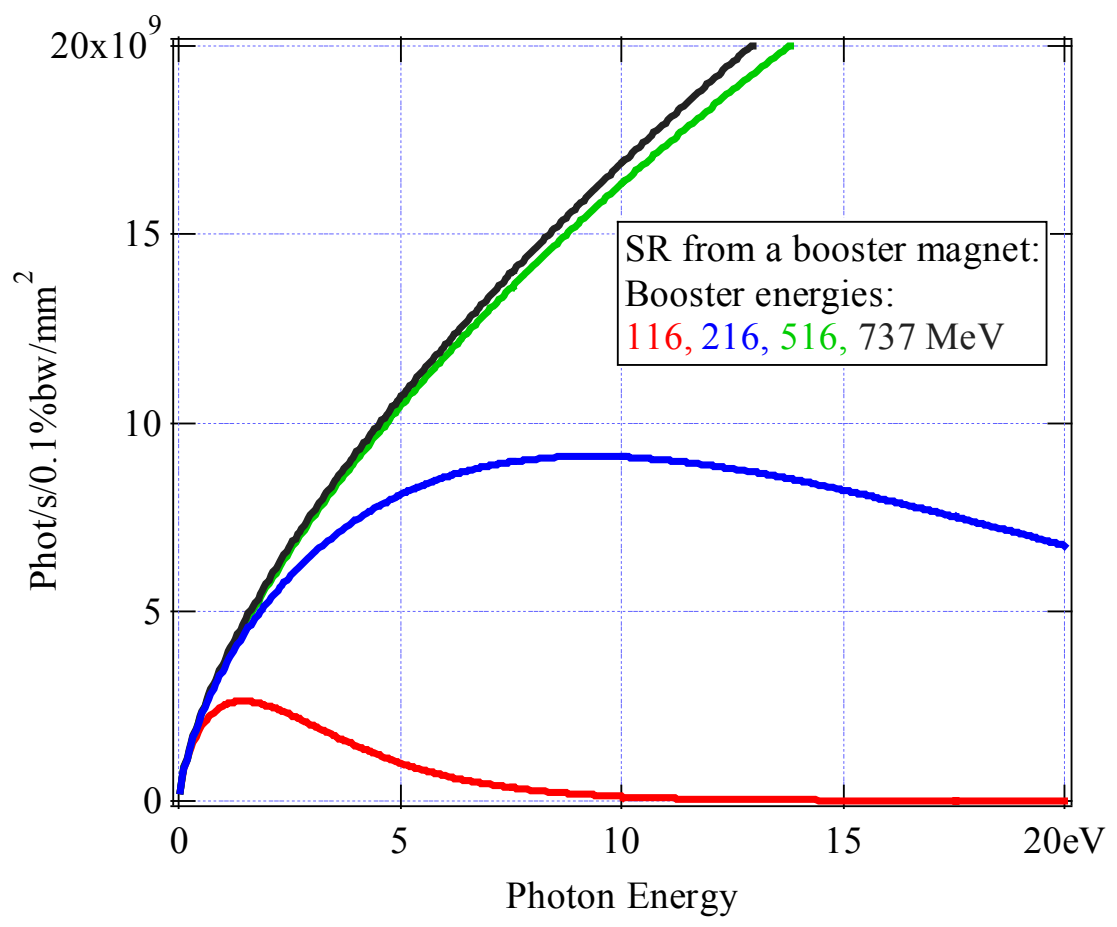

Figure 3. Spectra of synchrotron radiation from the booster bend magnet at different energies.

\section{System resolution budget}

One of the most important issues for the imaging system is its resolution. We can neglect geometrical aberrations because the optical system is very close to paraxial. The chromatic aberrations are eliminated by usage of the narrowband optical filter.

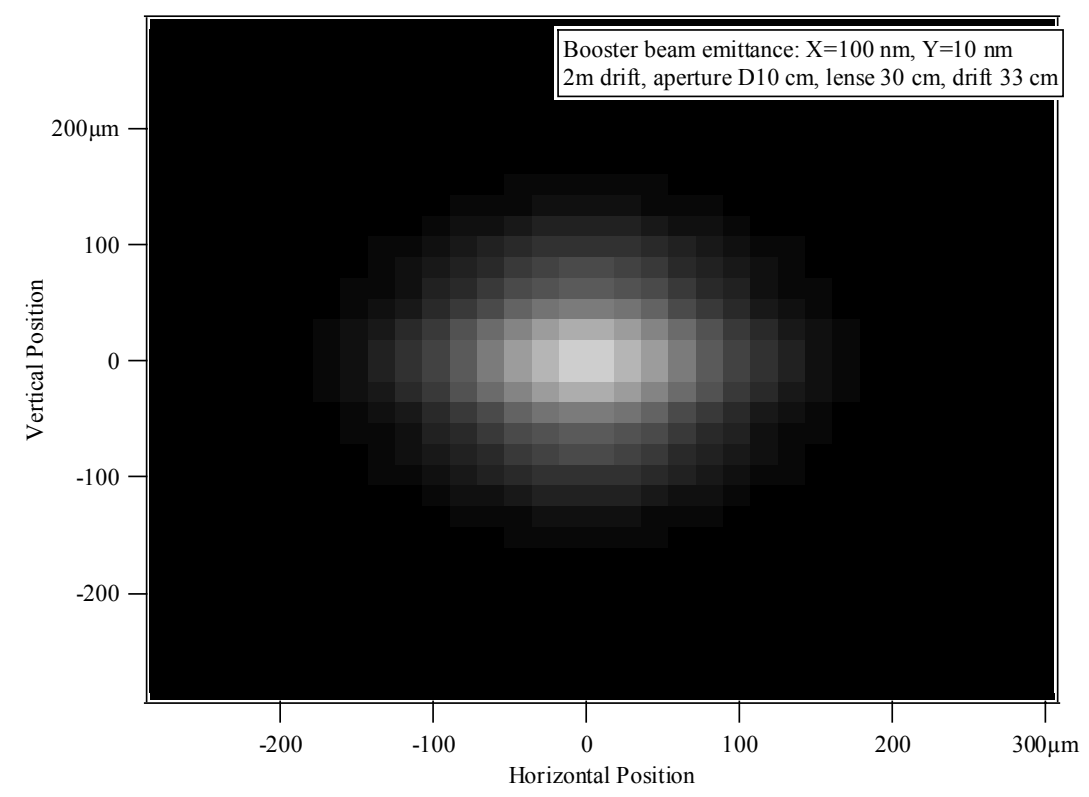

Figure 4. Calculated beam profile in the plane of camera sensor.

The actual beam size 5.7 times larger. 
An optical system, where source is at distance $L$ from the lens with focal length of $f$, creates image at distance $l=\frac{L f}{L-f}$. An aperture with diameter $d$ will create divergence due to the diffraction with angle $\alpha=1.22 \lambda / d$, which will define resolution in the image plane $\sigma_{\text {diff }}=\alpha \mathrm{l}=1.22 \lambda \mathrm{l} / \mathrm{d}$. For 1 " mirror tilted by $45^{\circ}$ effective $\mathrm{d}=17.8 \mathrm{~mm}$ and resolution in the CCD plane will be 15 microns.

The observation wavelength $\lambda=620 \mathrm{~nm}$ is longer then critical wavelength at extraction energy. Therefore, the radiation forming the image of the beam will have opening angle of $\sigma_{\gamma}{ }^{\prime}= \pm 0.41(\lambda / \rho)^{1 / 3}$, where $\rho=1.91 \mathrm{~m}$ is radius of curvature of the orbit in the NSLS booster. Substituting the values gives opening angle of $2.8 \mathrm{mrad}$ and radiation formation length $z=0.82 \lambda^{1 / 3} \rho^{2 / 3}$ equal to $11 \mathrm{~mm}$. The images of the electron beam at different parts of the trajectory will be focused at different locations and the image length will be $z^{\prime} \approx z \frac{f^{2}}{(L-f)^{2}}$. Resolution due to the non-zero source length can be estimated using demagnification factor $\sigma_{\text {source }}=5.7 \sigma_{\gamma}{ }^{\prime} z^{\prime} / 2$ and is 2.7 microns. The obtained value is much smaller then the diffraction resolution and could be neglected. The numerically calculated profile of the image of the electron beam is shown in Fig. 4.

The BLH201 CCD camera manufactured by Cohu has a sensor with 1/3" diagonal and number of pixels $768 \times 494$ (pitch of $8.8(\mathrm{H}) \times 10(\mathrm{~V})$ microns). Matrox Meteor-II 8-bit frame grabber produces image of $640 \times 480$ pixels, and for analyses we used pixel pitch of 10.5 microns for both directions. This value is smaller than optical system resolution and does not significantly affect overall system resolution.

Another factor affecting resolution is shift of the CCD sensor from the image plane. To preserve system resolution, camera should be positioned with accuracy better than $0.1 \mathrm{~mm}$. For this purpose it will be mounted on a translation stage with micrometer. The position will be scanned for the smallest observed beam size.

\section{Data Processing}

The digitized video camera signal is used for processing. The software allows capturing of consecutive frames after trigger synchronized with the injection impulse. Images, saved as a bitmap file on the hard drive, are analyzed for extraction of needed information. Presently, the following functions are realized: background subtraction, locating of the beam center for the orbit observation, transverse sizes measurement for the emittance estimations and detection of the beam instabilities, tilt angle calculation. The last function is needed because optical system rotates the beam image by approximately $30^{\circ}$. The fitted Gaussian profiles are displayed on the screen as blue dashed lines (Fig. 5), when the measured density distributions are shown as blue solid lines. Good match between fit and experimental data is observed. 


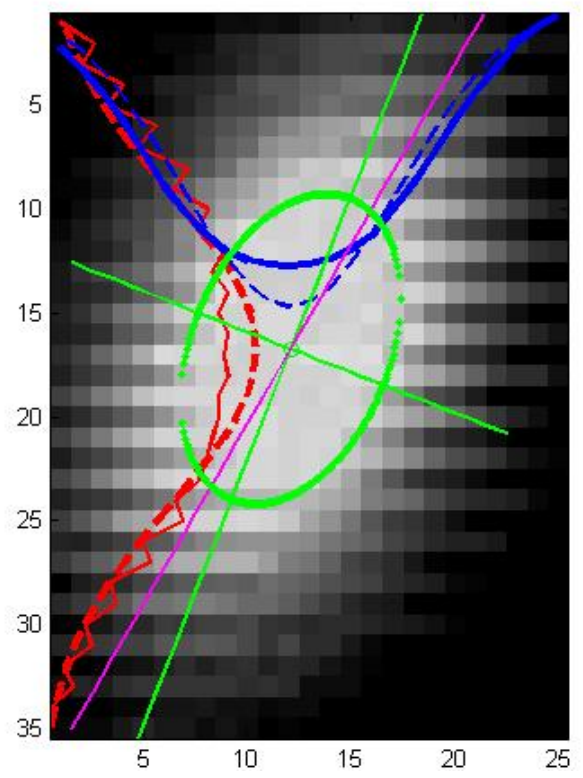

Figure 5. Processed image of the electron beam. Magenta lines show tilt of the optical system. Green lines show the ellipse axes and are used for calculation of coupling.
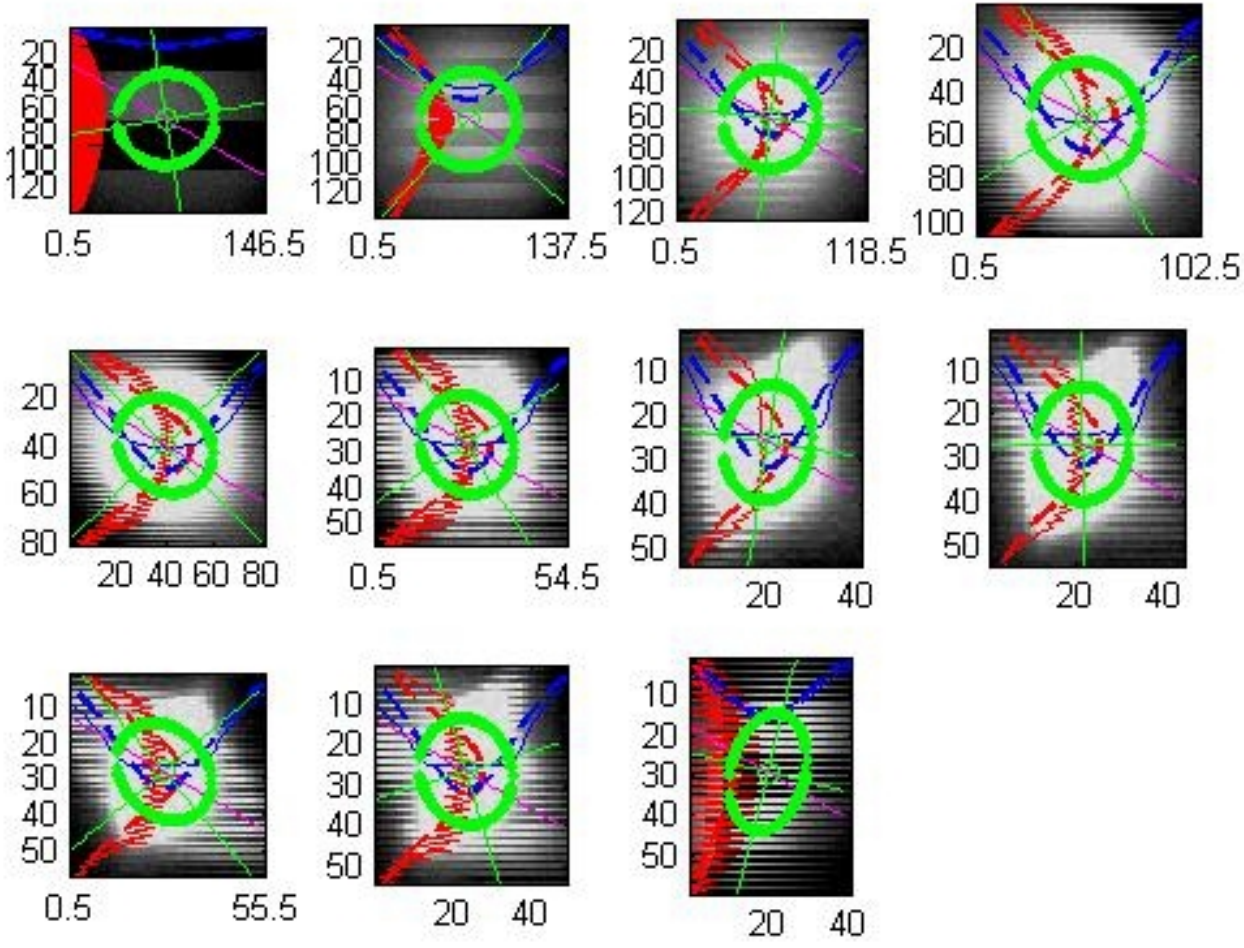

Figure 6. Consecutive frames of the electron beam circulating in the booster separated by $33 \mathrm{~ms}$. The image at the upper left corner correspond to injection and the utmost right image in the bottom row corresponds to extraction. 

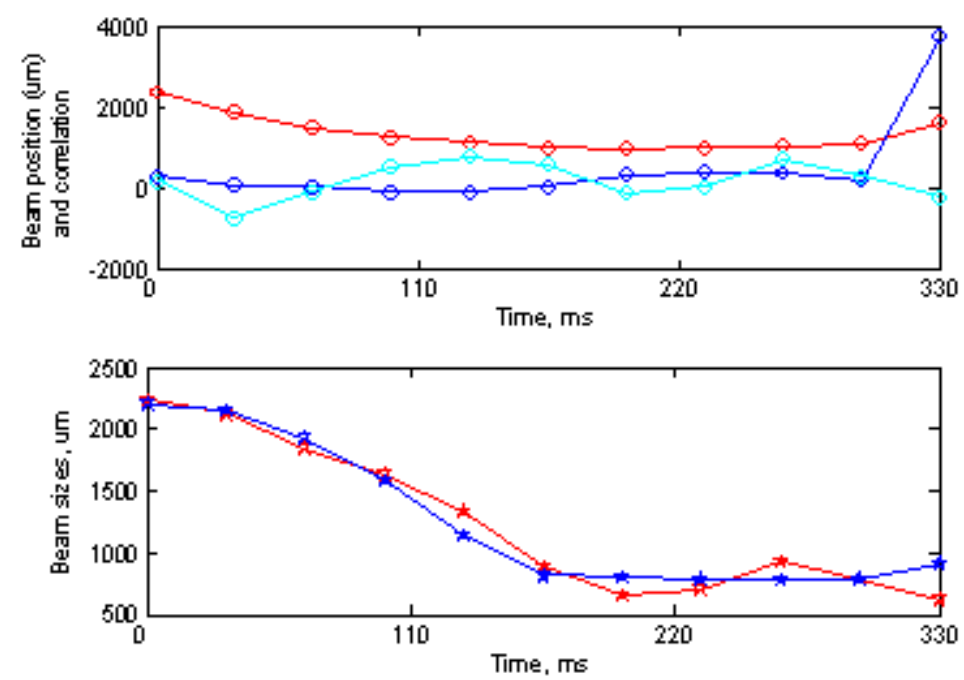

Figure 7. Parameters of the electron beam during ramp. Injection occurs at zero and extraction follows $\mathbf{3 2 0}$ milliseconds later. Actual injection and extraction time can vary because camera is not synchronized.

The dependence of the observed beam parameters (the horizontal and vertical sizes, the horizontal and vertical positions, and correlation) is shown in Fig. 6.

\section{Further improvements}

As a part of the upgrade from the present SRM set-up we are planning to install two beam splitters, which will provide light for position sensitive detector (PSD) and camera with external trigger capabilities. In order to obtain high resolution this camera will be also mounted on the translation stage. 